\title{
HSP90: A Novel Target Gene of miRNA-628-3p in A549 Cells
}

\author{
Jieli Pan, ${ }^{1}$ Fusheng Jiang $\mathbb{D},{ }^{2}$ Jia Zhou, ${ }^{3}$ Dehong Wu, ${ }^{4}$ Zhenhua Sheng, ${ }^{1}$ and Meiya Li $\mathbb{D}^{1}$ \\ ${ }^{1}$ Academy of Chinese Medical Sciences, Zhejiang Chinese Medical University, Hangzhou 310053, China \\ ${ }^{2}$ College of Life Science, Zhejiang Chinese Medical University, Hangzhou 310053, China \\ ${ }^{3}$ College of Basic Medical Sciences, Zhejiang Chinese Medical University, Hangzhou 310053, China \\ ${ }^{4}$ The Second Affiliated Hospital of Zhejiang Chinese Medical University, Hangzhou 310053, China
}

Correspondence should be addressed to Meiya Li; lmeiya@126.com

Received 19 March 2018; Accepted 12 April 2018; Published 20 May 2018

Academic Editor: Valli De Re

Copyright (C) 2018 Jieli Pan et al. This is an open access article distributed under the Creative Commons Attribution License, which permits unrestricted use, distribution, and reproduction in any medium, provided the original work is properly cited.

Lung cancer is one of the leading causes of cancer-related death in the world. MicroRNA- (miR-) 628-3p plays critical roles in many cancers, including lung cancer. We investigated how miR-628-3p affected migration and apoptosis in A549 cells. We used bioinformatics algorithms to predict the miR-628-3p target gene to study the molecular mechanism by which miR-628-3p contributes to lung cancer. Then, we used the luciferase reporter assay to identify whether heat shock protein 90a (HSP90) is a direct target of miR-628-3p. Western blotting and quantitative real-time PCR showed that miR-628-3p downregulated HSP90a protein expression via a posttranscriptional mechanism. We confirm that miR-628-3p promotes apoptosis and inhibits migration in A549 cells by negatively regulating HSP90. Our results may reveal a novel strategy for lung cancer treatment.

\section{Introduction}

Lung cancer is the most common cancer in the world and is the leading cause of cancer-related deaths [1]. The occurrence and development of lung cancer are a complex, multistep, and multilevel process involving multiple factors, and the mechanism involved remains unknown. Lung cancer has become a challenging topic and hotspot in tumor research. Although research on the early diagnosis and treatment of lung cancer in recent years has become more in depth, the overall prognosis and survival of patients with lung cancer are unsatisfactory; therefore, there is still much to be done in lung cancer research and treatment.

There are many types of lung cancer, among which nonsmall cell lung cancer (NSCLC) is the main type, accounting for about $75-80 \%$ of lung cancer cases [2]. As the symptoms are not apparent and detection is difficult at stage I and II NSCLC, it is mostly diagnosed at the late stages, which reduces the five-year survival rate. Therefore, earlier detection of lung cancer is needed and could lead to more effective management of the disease.

There are a variety of abnormal factors in lung cancer $[3,4]$; research into the mechanism of action has been performed at molecular level, but the exact molecular mechanism remains unclear. With the development of molecular biology technology, the discovery of microRNAs (miRNAs) has opened up a new avenue in lung cancer research and provides new ideas and approaches for diagnosing and treating lung cancer.

miRNAs are a family of naturally occurring, small, endogenous noncoding RNAs that are 18-25 nucleotides in length [5]. miRNAs influence many biological processes, including cell proliferation, apoptosis, development, differentiation, migration, and survival. miRNAs are differentially expressed in cancer and play important roles in regulating gene expression by base-pairing to the complementary sites on the target mRNAs, consequently blocking translation or triggering the degradation of the target mRNAs $[6,7]$. Growing evidence has demonstrated the role of miRNAs in many human tumors, including breast [8], ovarian [9], pancreatic [10], thyroid [11], and lung cancers [1].

The altered expression of tissue miRNAs has been associated with many diseases, particularly cancer. In recent years, the role of $\mathrm{miR}-628-3 \mathrm{p}$ in cancer has received increased attention. miR-628-3p is abnormally expressed in many cancers, such as colon [12], gastric [13], and pancreatic cancer [14]. The most recent study, which performed TaqMan low-density DNA microarray comparative analysis of more than 200 cases 
TABLE 1: siRNAs against HSP90 gene.

\begin{tabular}{|c|c|c|}
\hline siRNA & Sequence & Target mRNA sequence \\
\hline \multirow{2}{*}{ siRNA 1} & F: $5^{\prime}$ - GCCCUAAGAGACAACUCAAdTdT- $3^{\prime}$ & $5^{\prime}$-GCCCTAAGAGACAACTCAA-3 ${ }^{\prime}$ \\
\hline & R: $3^{\prime}$ - dTdTCGGGAUUCUCUGUUGAGUU-5' & \\
\hline \multirow{2}{*}{ siRNA 2} & F: $5^{\prime}$ - GGAACGUGAUAAAGAAGUAdTdT-3' & $5^{\prime}$-GGAACGTGATAAAGAAGTA-3' \\
\hline & R: $3^{\prime}$ - dTdTCCUUGCACUAUUUCUUCAU-5' & \\
\hline \multirow{2}{*}{ siRNA 3} & F: $5^{\prime}$ - GCACCAGAAUGAAGGAGAAdTdT-3' & $5^{\prime}$-GCACCAGAATGAAGGAGAA- $3^{\prime}$ \\
\hline & R: $3^{\prime}$ - dTdTCGUGGUCUUACUUCCUCUU- $5^{\prime}$ & \\
\hline
\end{tabular}

of early lung cancer and more than 170 cases of normal human plasma samples, found abnormal high expression miR-628$3 p$ in the lung cancer cases, suggesting that miR-628-3p can be used as a marker of early diagnosis of lung cancer [15]. However, the regulatory mechanism of miRNA in lung cancer is not clear, and more research is needed.

At present, numerous clinical studies have confirmed that the remission rate of molecular targeted drugs in patients with lung cancer is not very high, and drug resistance against targeted drugs is increasing. Therefore, further study of the main target in lung cancer is imperative, and developing lung cancer treatment closely related to miRNA-target research has gradually become a hot research topic. Identifying the target of miR-628-3p and clarifying the mechanism of miR628-3p would provide new ideas and methods for diagnosing and treating lung cancer.

Heat shock protein 90a (HSP90a), one of the most abundant and conserved chaperone proteins, aids in preserving the integrity and function of numerous client proteins. Plasma HSP90a protein levels are useful as a diagnostic biomarker in lung cancer [16].

In this study, we investigated the relationship between miR-628-3p and HSP90a in A549 lung cancer cells. Moreover, we provide evidence that HSP90 is a target gene of miR-628$3 \mathrm{p}$ in A549 cells.

\section{Materials and Methods}

2.1. Cell Culture. 293T and A549 cells were cultured in Dulbecco's modified Eagle's medium (Invitrogen, USA) supplemented with $10 \%(\mathrm{v} / \mathrm{v})$ fetal bovine serum (Gibco, USA) and $1 \%$ antibiotics $(100 \mathrm{U} / \mathrm{mL}$ penicillin and $100 \mathrm{mg} / \mathrm{L}$ streptomycin sulfate). All cells were grown in a humidified atmosphere at $37^{\circ} \mathrm{C}$ with $5 \% \mathrm{CO}_{2}$.

2.2. Oligonucleotide Transfection. miR-628-3p mimics and HSP90 small interfering RNAs (siRNAs) were designed and synthesized by RiboBio (Guangzhou, China); Table 1 lists their sequences and the targeted mRNAs.

Cells were plated on 6-well plates (Corning, USA) and cultured overnight to $40-60 \%$ confluence. Transfection was performed with Lipofectamine 3000 (Invitrogen, USA) according to the manufacturer's protocol. After $48 \mathrm{~h}$, we harvested the cells and detected HSP90 and miR-628-3p by western blotting and quantitative real-time polymerase chain reaction (qRT-PCR), respectively.

2.3. Apoptosis Assay. Apoptosis was determined by annexin $\mathrm{V}$ and propidium iodide (PI) staining using an apoptosis detection kit (556547, BD) according to the manufacturer's instructions. Briefly, A549 cells were transfected with $50 \mathrm{nM}$ NC mimic, miR-628-3p mimic, or HSP90 siRNAs for $48 \mathrm{~h}$. Then, the cells were washed with $50 \mathrm{mmol} / \mathrm{L}$ cold phosphate buffer ( $\mathrm{pH} 7.5$ ), centrifuged at $1200 \times \mathrm{g}$ for $5 \mathrm{~min}$, and suspended in binding buffer.

The treated cells were incubated with annexin $\mathrm{V}$ and PI for $15 \mathrm{~min}$ at room temperature and then analyzed for annexin $\mathrm{V}$ binding affinity within $1 \mathrm{~h}$ by flow cytometry (BD Accuri C6).

2.4. RNA Extraction and $q R T-P C R$. Total RNA from the cells was extracted using TRIzol (Invitrogen) according to the manufacturer's protocol. Complementary DNA (cDNA) was synthesized from $1 \mu \mathrm{g}$ total RNA with a PrimeScript reverse transcription (RT) reagent kit with genomic DNA (gDNA) Eraser (TaKaRa, China) in a $20 \mu \mathrm{L}$ volume [mRNA genes, RT primers; miRNA, U6 rRNA, and miRNA-specific primers (Bulge-Loop miRNA quantitative PCR (qPCR) primers, RiboBio)]. Real-time PCR was carried out with SYBR Green I mix reagents (TaKaRa, Dalian, China) in a $20 \mu \mathrm{L}$ reaction volume $(10 \mu \mathrm{L}$ SYBR Green I mix, $200 \mathrm{mM}$ forward and reverse primer, $1 \mu \mathrm{L}$ cDNA template) on a 7500 Real-Time PCR System (Applied Biosystems). Each reaction was run in triplicate; the threshold cycle $(\mathrm{Ct})$ data were determined by fixed threshold settings, and the average Ct of triplicate PCR was calculated. The relative miRNA levels were confirmed using the comparative Ct method. The amount of internal control U6-related miRNA was calculated using the equation $2^{-\Delta \Delta \mathrm{Ct}}$, in which $\Delta \Delta \mathrm{Ct}=(\mathrm{Ct}$ miRNA $-\mathrm{Ct} \mathrm{U} 6)$ tumor $-(\mathrm{Ct}$ miRNA - Ct U6) control. The relative amount of HSP90 mRNA was normalized to 18s rRNA using a method similar to the one described above.

2.5. Protein Extraction. Cells were washed three times with phosphate-buffered saline (PBS) chilled to $4^{\circ} \mathrm{C}$. Whole-cell proteins were extracted with M-PER Mammalian Protein Extraction Reagent (78503, Thermo Fisher Scientific, USA) containing protease and phosphatase inhibitor (Roche, Germany) at $4^{\circ} \mathrm{C}$ for $30 \mathrm{~min}$. Then, the samples were centrifuged at $14,000 \times \mathrm{g}$ for $10 \mathrm{~min}$, and the supernatant was transferred to a new tube for analysis.

2.6. Automated Western Immunoblotting. Before blotting, the protein was quantified using the bicinchoninic acid (BCA) method. Simple western immunoblotting was performed on a Peggy Sue system (ProteinSimple, California, USA) using a Size Separation Master Kit with Split Buffer $(12-230 \mathrm{kDa})$ according to the manufacturer's standard instruction and 
using anti-HSP90 (4877S, CST, USA) and anti- $\beta$-actin (4970S, CST, USA) antibodies. Compass software (version 2.7.1, ProteinSimple) was used to program the Peggy Sue and for presentation (and quantification) of the western immunoblots. Output data were displayed from the softwarecalculated average of seven exposures (5-480 s).

2.7. Luciferase Reporter Assay. We used luciferase reporter assays to verify whether miR-628-3p directly targets the HSP90 gene and used a pHY-REPORT system (Hanyin Biotechnology, Shanghai, China). Briefly, 46-mer doublestranded oligonucleotides containing the predicted miRNA binding sites in HSP90 were synthesized and ligated between the XhoI and BamHI restriction sites of the pHY-REPORT luciferase vector to establish the pLUC-HSP90 vector.

A549 cells were cotransfected with $50 \mathrm{nM}$ miR-628-3p mimic or negative control (NC) mimic in 96-well plates containing $200 \mathrm{ng} / \mu \mathrm{L}$ pLUC-HSP90 or pLUC-mutHSP90 plasmid and $10 \mathrm{ng}$ Renilla luciferase (internal transfection efficiency control). After 24-h transfection, luciferase activity was detected using a Dual-Luciferase Reporter Assay System (Promega, USA) according to the manufacturer's protocol. Luminescence intensity was read with a microplate luminometer using the corresponding Promega protocol. Transfections were performed in duplicate and repeated three times.

2.8. Wound Healing Assay. An in vitro wound healing assay was performed to measure the unidirectional migration of A549 cells. A549 cells $\left(10 \times 10^{4}\right.$ cells/mL) were seeded in 6well plates and were allowed to grow for $24 \mathrm{~h}$ after being transfected with miR-628-3p mimic, NC mimic, or HSP90 siRNAs. Then, the A549 monolayers were scratch-wounded in a straight line using a $10 \mu \mathrm{L}$ pipette tip when cells were $70-80 \%$ confluent. Immediately after wounding and after $24 \mathrm{~h}$ incubation, the cells were photographed under a microscope (Axio Observer A1, Zeiss, Germany). Migration was calculated as the area of A549 cells that had migrated from the injured edge in to the wound zone. At least four points in each of three random fields were examined for each of three independent wounds. The wound closure (\%) was calculated as migrated cell surface area/total surface area $\times 100$.

2.9. Statistical Analysis. All data are presented as the mean \pm SEM and were analyzed using SPSS16 software. $p$ values were calculated using Student's unpaired $t$-test (for two groups) or $Z$-way analysis of variance (for more than two groups). Statistical significance was taken at $p<0.05$ and $p<0.001$.

\section{Results}

3.1. Screening of miR-628-3p Target Genes. TargetScan (http:// www.targetscan.org/mamm_31/) and NCBI (https://www.ncbi .nlm.nih.gov/) were used to analyze the information of multiple genes that can bind with miR-628-3p, and we selected nine genes with a high degree of integration: FAS, APAF1, XIAP, HSP90, PIK3R3, RIPK1, CASP3, PMAIP1, and AKT2.

To further screen target genes, miR-628-3p mimic was transfected into 293T cells, and quantitative RT-PCR (qRTPCR) was used to detect the expression levels of the predicted

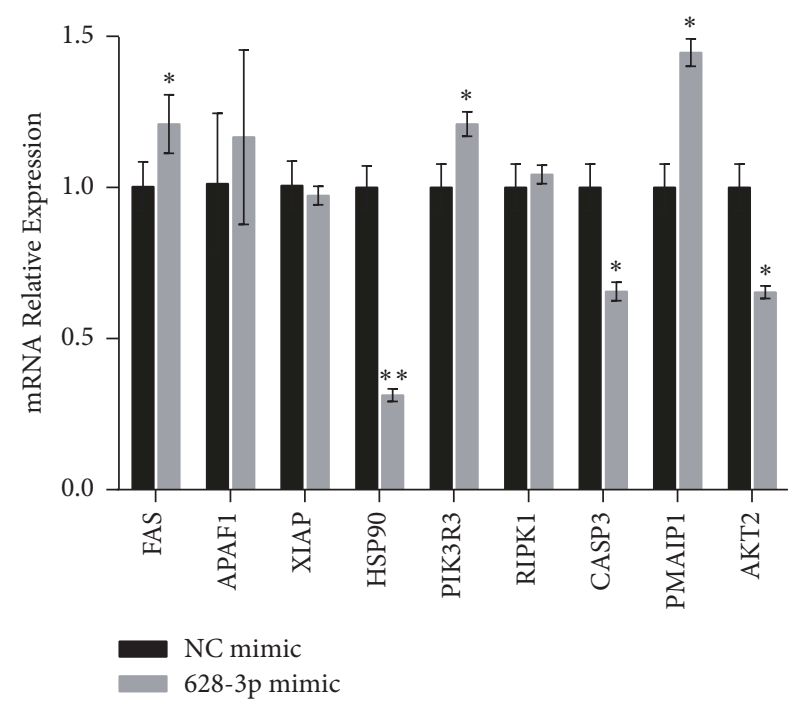

FIGURE 1: miR-628-3p and target gene expression in 293 T cells. ${ }^{*} p<$ $0.05,{ }^{* *} p<0.001$.

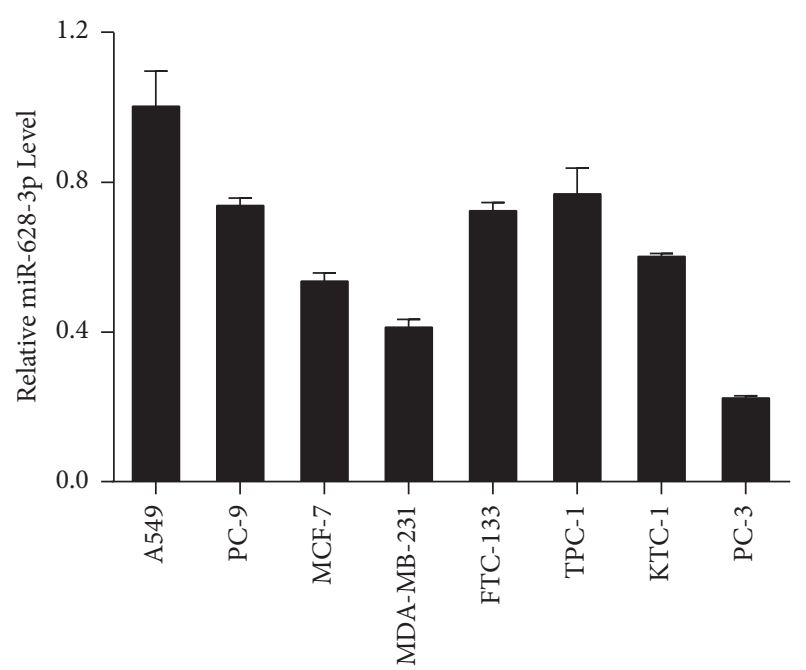

FIGURE 2: miR-628-3p is expressed differentially in different cancer cells. Relative levels of miR-628-3p in A549 cells were normalized against that of the other cell lines. $n=5$, mean \pm SD.

genes as regulated by miR-628-3p. Figure 1 shows that FAS, PIK3R3, and PMAIP1 were upregulated after miR-628$3 \mathrm{p}$ mimic transfection, while HSP90, CASP3, and AKT1 were downregulated; among the three downregulated genes, HSP90 was significantly downregulated, so it was selected for further research.

3.2. miR-628-3p Was Expressed High in A549 Cells. We used eight cancer cell lines (A549, PC-9, MCF-7, MDA-MB-231, FTC-133, TPC-1, KTC-1, and PC-3) to select a cell line in which to research the regulatory relationship between HSP90 and miR-628-3p. miR-628-3p expression levels in the cell lines were compared using qRT-PCR. As miR-628-3p expression was higher in A549 cells than in the other cancer cells (Figure 2), we selected the A549 cell line for further research. 


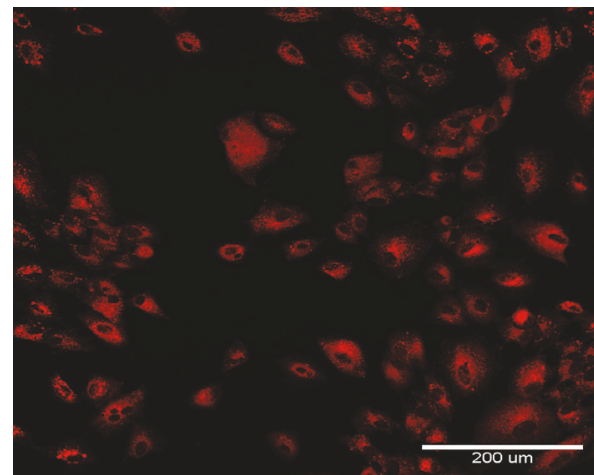

(a1)

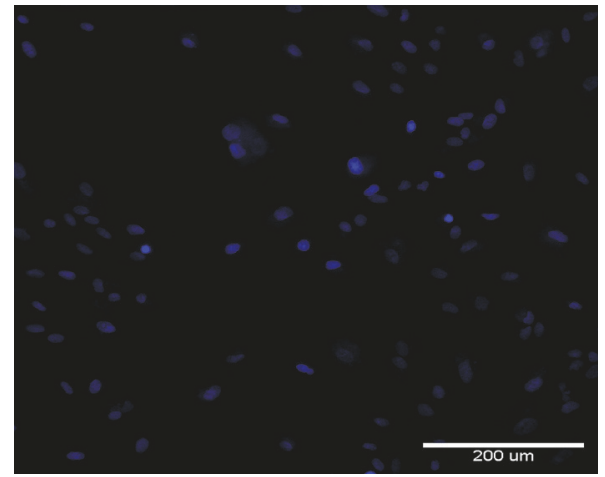

(a2)

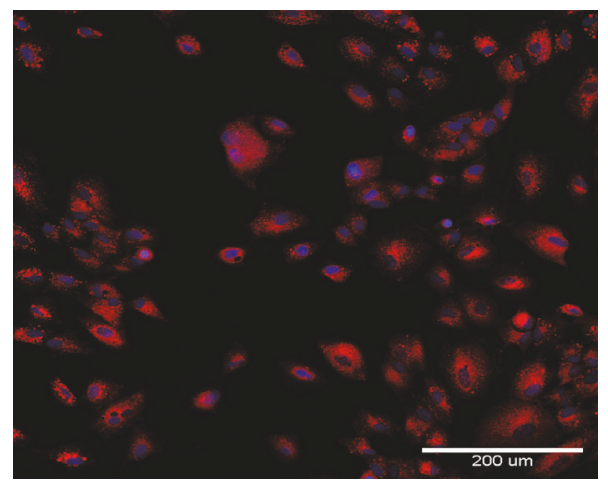

(a3)

(a)



(b)

Figure 3: (a) Immunofluorescence images of A549 cells transfected with miR-628-3p. (b) Relative miR-628-3p expression levels after transfection in A549 cells. ${ }^{* *} p<0.001$.

3.3. The Expression of miR-628-3p Was Efficient after Transfection in $A 549$ Cells. Cy3 was used as an indicator to verify the vector transfection efficiency. After 24-h transfection, the cells were photographed by fluorescence microscopy, and each cell emitted red fluorescence (Figure 3(a)), indicating that vector transfection efficiency was very high.
To verify miR-628-3p expression, A549 cells were transfected with miR-628-3p mimic or NC mimic (vector control); the cells that were not transfected with vector were used as the blank control. miR-628-3p expression was detected by fluorescence qPCR (Figure 3(b)), which showed that cells transfected with miR-628-3p mimic had 


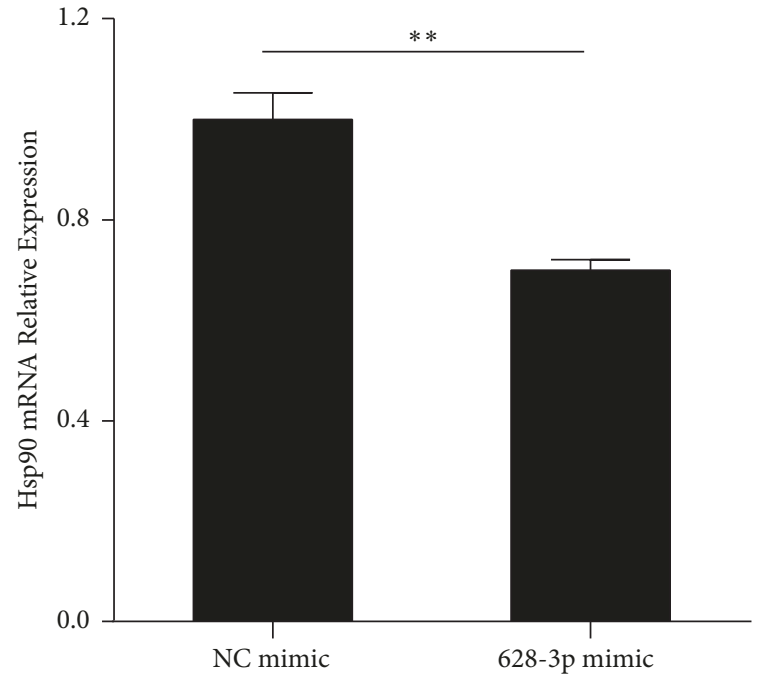

(a)



(b)

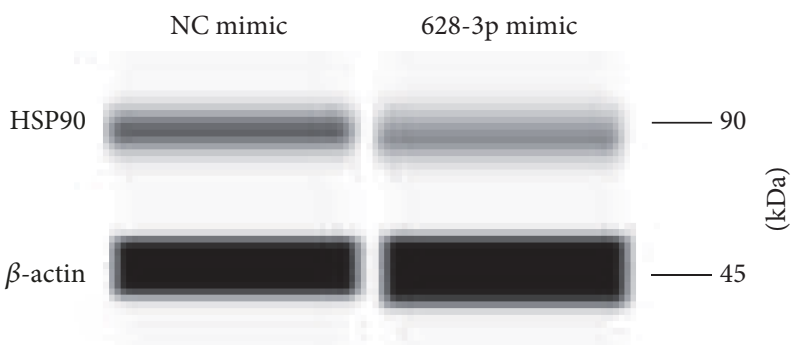

(c)

FiguRE 4: (a) HSP90 mRNA was downregulated in A549 cells transfected with miR-628-3p mimic. The average mRNA expression from the NC group is designated as 1. (b, c) Western blot measurement of HSP90 protein levels after 48-h transfection with miR-628-3p mimic. ${ }^{* *} p<0.001$.

far higher miR-628-3p expression than the control, indicating that miR-628-3p mimic expression was very efficient.

3.4. HSP90 Protein Was Downregulated in A549 Cells Transfected with miR-628-3p Mimic. Western blotting was used to analyze the regulatory relationship between miR-628-3p and HSP90 in A549 cells following transfection with miR$628-3 p$ mimic or NC mimic. Immunoblotting with antibodies against HSP90 and $\beta$-actin showed that miR-628-3p obviously inhibited HSP90 expression (Figure 4), indicating a negative regulatory relationship between miR-628-3p and HSP90.

3.5. miR-628-3p Regulated HSP90 Expression by Targeting Its $3^{\prime}$ UTR. Luciferase assay was used to confirm whether the $3^{\prime}$ untranslated region ( $3^{\prime}$ UTR) of the predicted target genes had putative miR-628-3p-binding sites. HSP90 was selected as a potential target gene of miR-628-3p. TargetScan predicted the consequential pairing of the HSP90 $3^{\prime}$ UTR and miR-628-3p (Figure 5(a)), where the dual-luciferase reporter assay showed that miR-628-3p directly bound to the HSP90 $3^{\prime}$ UTR in A549 cells. To test the HSP90 putative binding site, we generated a luciferase reporter (pLUC-HSP90) and a mutant HSP90 construct (pLUCmutHSP90) in which the HSP90 $3^{\prime}$ UTR was altered using a site-directed mutagenesis kit. miR-628-3p significantly regulated the luciferase activity of the HSP90 construct (Figure 5(b)), whereas the mutant construct did not generate luciferase activity. These results indicate that miR628-3p may regulate HSP90 expression by targeting its $3^{\prime}$ UTR.

\section{6. siRNA Transfection Interfered with Downregulated HSP90} Expression. Three siRNAs targeting the HSP90 gene were transfected into the A549 cells, and the relative mRNA expression was measured $48 \mathrm{~h}$ after transfection. siRNA2 is better than the other two siRNAs (Figure 6(a)). HSP90 protein expression was detected by western blotting; siRNA2 interference downregulated HSP90 expression significantly as compared to the other two siRNAs (Figures 6(b) and 6(c)) significantly. 


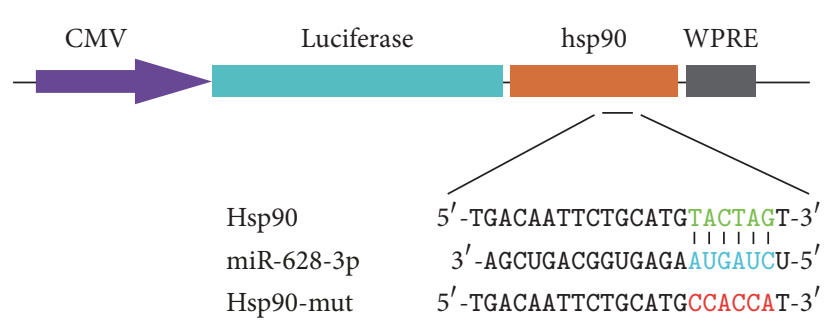

(a)

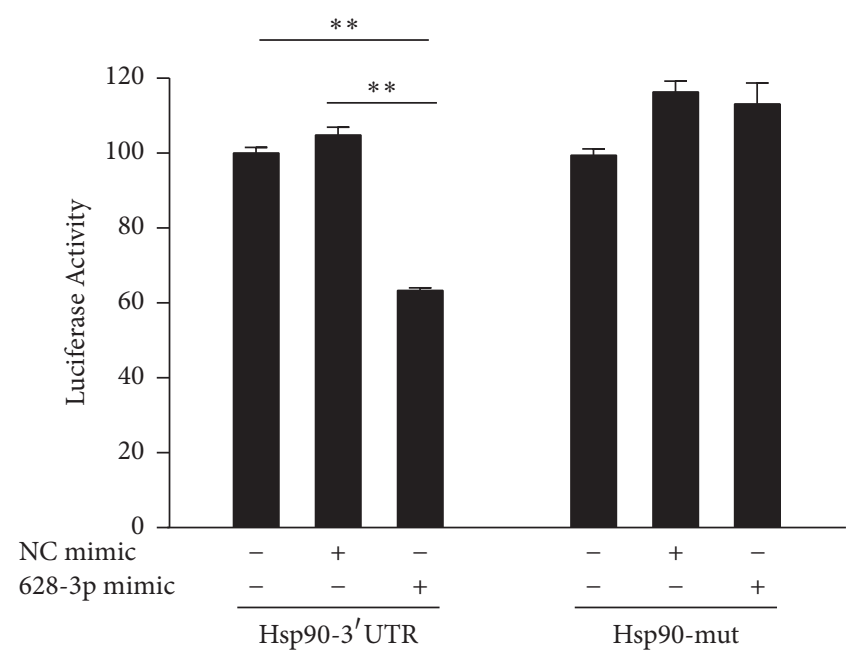

(b)

FIGURE 5: miR-628-3p directly regulates HSP90 expression in A549 cells by targeting the HSP90 3' UTR. (a) Predicted consequential pairing of HSP90 $3^{\prime}$ UTR and miR-628-3p by TargetScan. (b) Luciferase assay in A549 cells. pLUC-HSP90 vector was cotransfected with miR-628-3p mimic or NC mimic. Luciferase activity in pLUC-HSP90 A549 cells was significantly decreased following ectopic expression of miR-628-3p mimic. ${ }^{* *} p<0.001$.

3.7. miR-628-3p Induced Apoptosis in A549 Cell. The rate of apoptosis in the A549 cells was measured by flow cytometry with annexin $\mathrm{V}$ and PI staining following 48$\mathrm{h}$ transfection. Figures $7(\mathrm{a})$ and $7(\mathrm{~b})$ show the percentage of early or late apoptotic cells following transfection with NC mimic and miR-628-3p mimic, and Figures $7(\mathrm{c})$ and $7(\mathrm{~d})$ show the percentage of early or late apoptotic cells following transfection with the control and the three HSP90 siRNAs. Transfection with miR-628-3p mimic and the HSP90 siRNAs induced apoptosis in the A549 cells.

3.8. miR-628-3p Inhibited Migration in A549 Cells. To understand how miR-628-3p affects cell migration, A549 cells were transfected with HSP90 siRNAs or miR-628-3p mimic. Transfection with miR-628-3p mimic inhibited A549 cell migration (Figures 8 (a) and $8(\mathrm{~b})$ ), as did transfection with the HSP90 siRNAs (Figures $8(\mathrm{c})$ and $8(\mathrm{~d})$ ), suggesting that miR-628-3p overexpression and interference with HSP90 expression have the same effect of decreasing A549 cell migration.

\section{Discussion}

The 2018 US cancer statistics reported an estimated 1,735,350 new cases and 609,640 new deaths from lung and branches cancer in the United States, indicating that lung and branches cancer is the leading cause of death among all tumors in the world [17]. Although the development of diagnostic and prognostic techniques has improved the survival of patients with lung cancer, most patients are diagnosed at the late stage. miRNAs function as tumor suppressors or oncogenes in various human cancers, including lung cancer [18]. Therefore, identifying the tumorassociated miRNAs and their target genes that underlie lung carcinogenesis might reveal novel therapeutic targets.

Some studies have indicated that miRNAs could play an important role in the initiation and progression of lung cancer; for example, overexpression of miR-21 promotes radiation-resistance of NSCLC [19], miR-125b regulates human NSCLC cell apoptosis via the phosphatidylinositol 3-kinase/AKT/glycogen synthase kinase $3 \beta$ 




(a)



(b)



(c)

FiguRE 6: (a) qRT-PCR measurement of HSP90 relative expression after 48-h siRNA transfection. HSP90 mRNA was downregulated in the siRNA-transfected A549 cells. The average MIR628-3P mRNA expression from the NC group is designated as 1. (b, c) Western blot measurement of HSP90 protein after 48 -h transfection. HSP90 protein was downregulated in the siRNA-transfected A549 cells. ${ }^{* *} p<$ 0.001 .

(PI3K/AKT/GSK3 $\beta$ ) signaling pathway [20], and miR-9 regulates NSCLC cell invasion and migration by targeting eukaryotic translation initiation factor 5A2 (EIF5A2) [21]; in addition, miR-628-3p and miR-425-3p expression is significantly higher in early-stage lung cancer, and miR532 expression is significantly lower than in the other types [15]. However, how miR-628-3p functions in lung cancer and which gene it targets are not known. Based on the TargetScan prediction, we selected nine genes with high degree of integration that can bind with miR-628$3 \mathrm{p}$; among the three downregulated genes, AKT1 has an important role in lung carcinogenesis, and depletion of AKT1 has antiproliferative and antimigratory effects [22]. miR-9500 represses lung tumorigenesis and metastasis by targeting AKT1 [23]. CASP3 is a previously unidentified target of miR-137 and plays an essential role in miR-137mediated lung cancer progression [24]. HSP90 plays an essential role in maintaining cellular protein homeostasis by acting as a molecular chaperone to aid in the folding and intracellular trafficking of its protein clients [25]. The in silico prediction indicated that HSP90 was a potential target of miR-628-3p, and it was significantly downregulated by miR-628-3p, so HSP90 was selected for further study.

We found that siRNA silencing of HSP90 or HSP90 downregulation by miR-628-3p overexpression promoted A549 cell apoptosis and inhibited A549 cell migration. qRT-PCR and western blotting confirmed that miR-628-3p mimic transfection into A549 cells downregulated HSP90 expression, as did HSP90 siRNA transfection. In addition, 


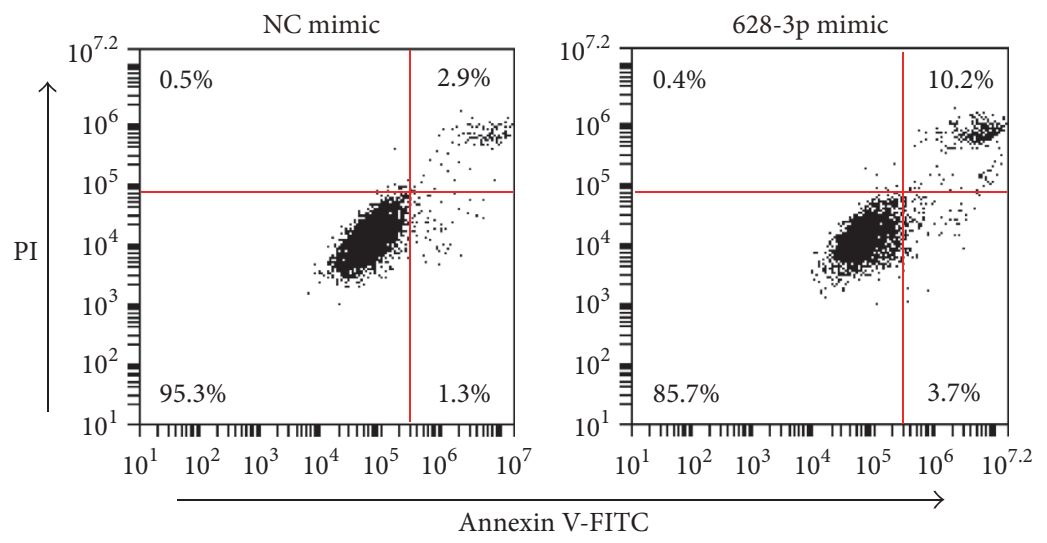

(a)

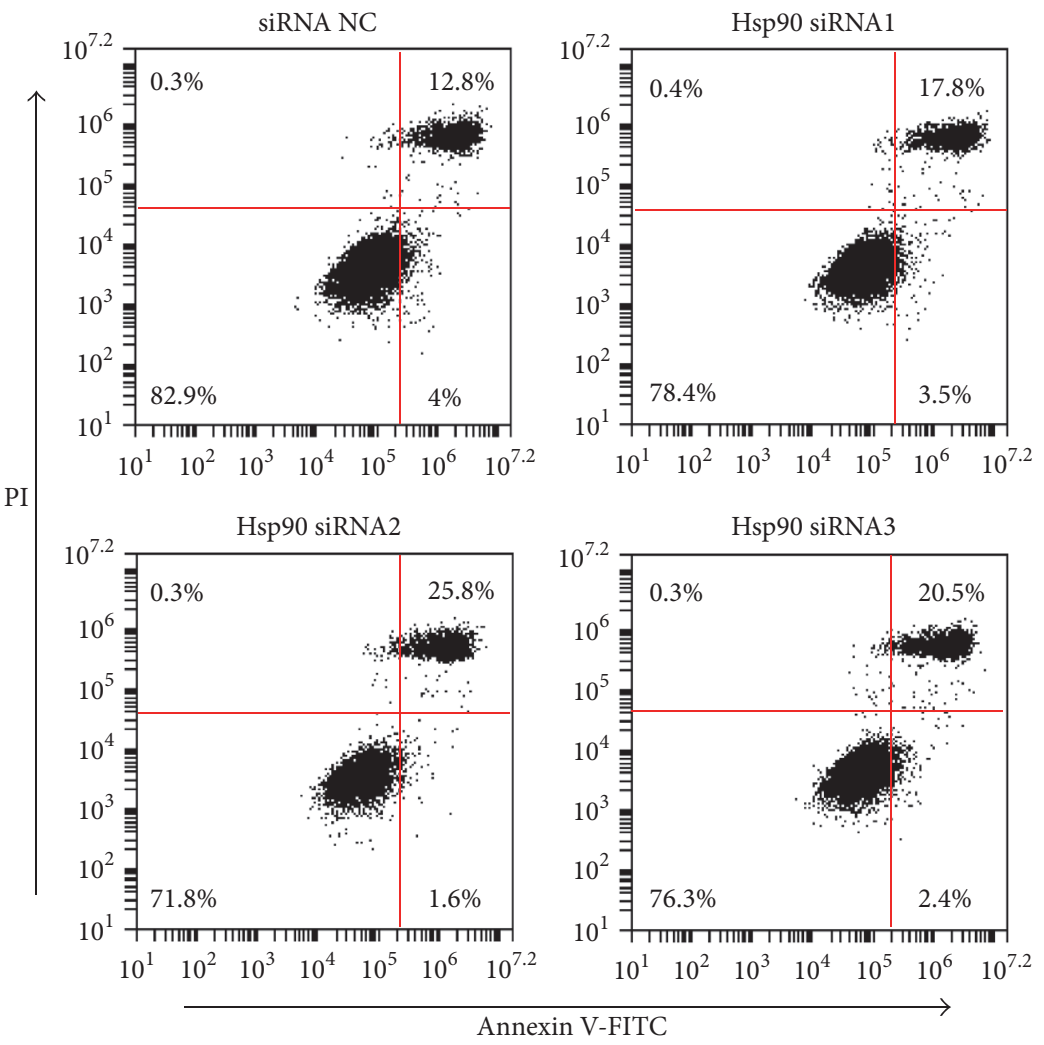

(c)

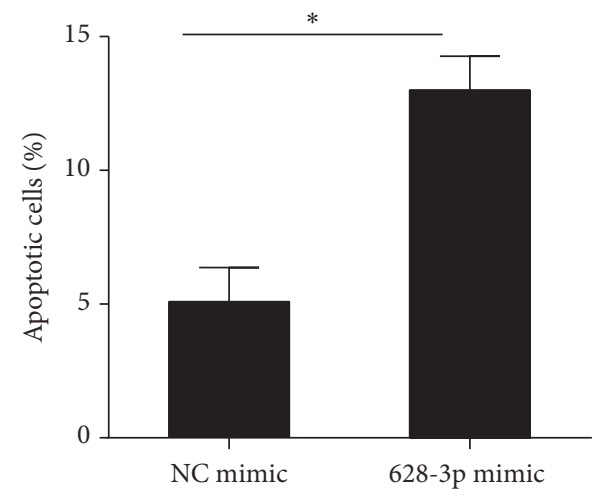

(b)

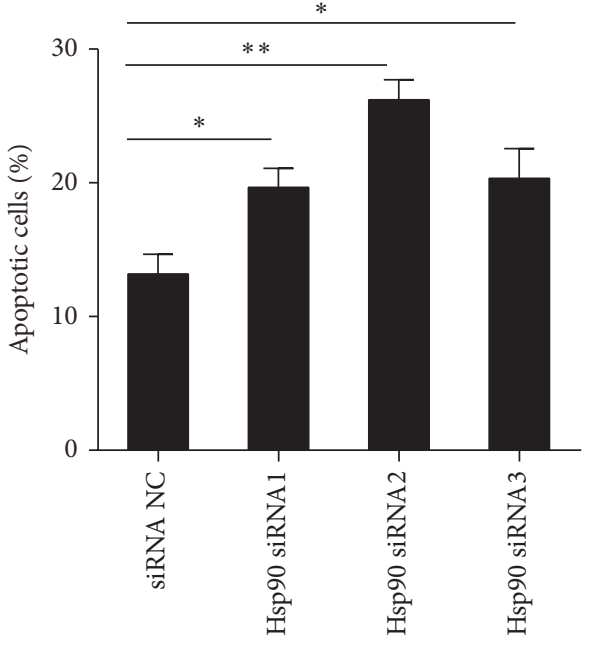

(d)

FiguRE 7: HSP90 expression levels affect A549 cell apoptosis. (a, b) miR-628-3p mimic transfection induced apoptosis in A549 cells. (c, d) HSP90 siRNA transfection induced apoptosis in A549 cells. ${ }^{*} p<0.05,{ }^{* *} p<0.001$.

the luciferase assay indicated that $\mathrm{miR}-628-3 \mathrm{p}$ may regulate HSP90 expression by targeting its $3^{\prime}$ UTR.

\section{Conclusion}

In summary, our results indicate that miR-628-3p promotes A549 cell apoptosis and inhibits A549 cell migration by negatively regulating HSP90. Further studies should consider the relationship between miR-628-3p and HSP90, which may reveal a novel therapeutic strategy for lung cancer.

\section{Data Availability}

The data used to support the findings of this study are available from the corresponding author upon request. 
(h)



(a)

(h)

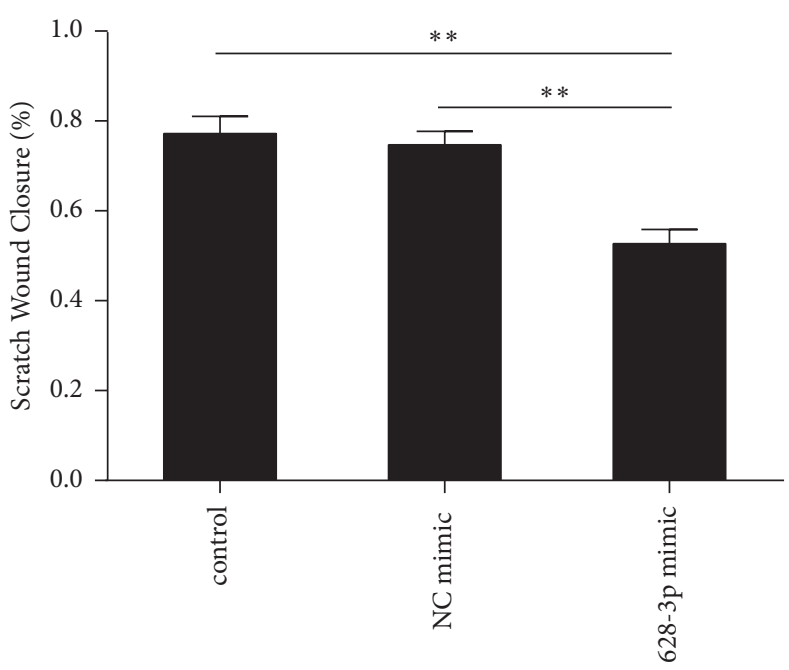

(b)

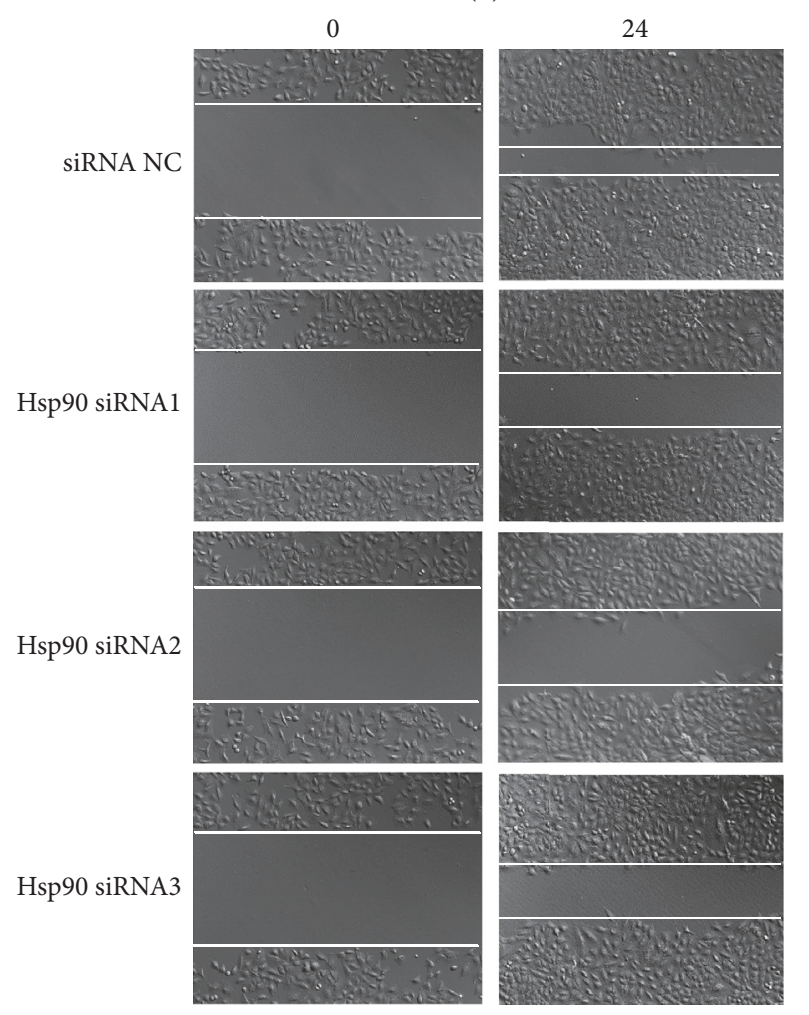

(c)

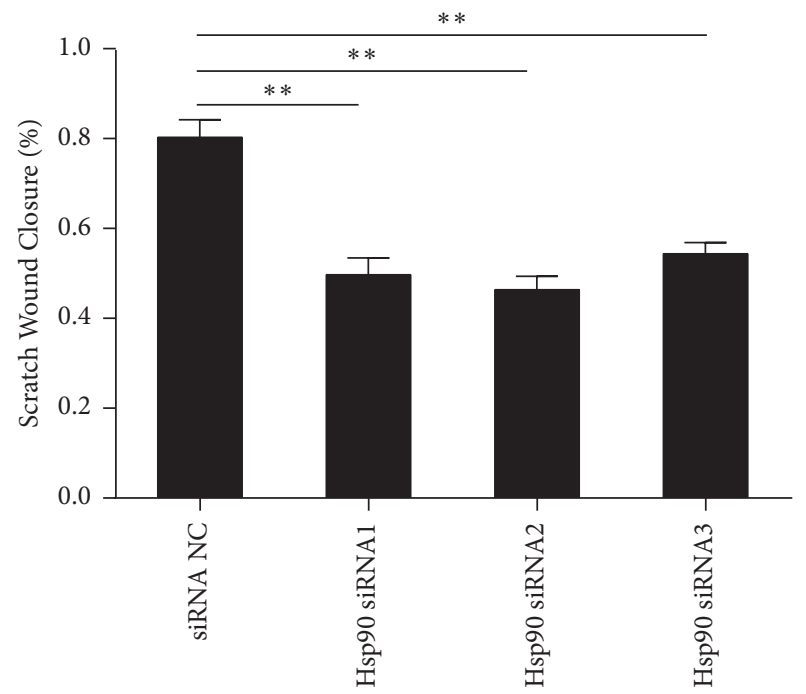

(d)

FIGURE 8: RNA interference of HSP90 inhibits wound healing. (a) A549 cells transfected with control, NC mimic, and miR-628-3p mimic photographed at $0 \mathrm{~h}$ and $24 \mathrm{~h}$ after wounding. (b) Scratch wound closure in A549 cells transfected with control, NC mimic, and miR-628-3p mimic. (c) A549 cells transfected with NC or HSP90 siRNAs photographed at $0 \mathrm{~h}$ and $24 \mathrm{~h}$ after wounding. (d) Scratch wound closure in A549 cells transfected with NC or HSP90 siRNAs. ${ }^{* *} p<0.001$. 


\section{Conflicts of Interest}

The authors declare that there are no conflicts of interest regarding the publication of this paper.

\section{Acknowledgments}

This work was funded by Science Foundation of Zhejiang Chinese Medical University (no. 2018ZZ12) and the Traditional Chinese Medicine Science Funding of Zhejiang Province (no. 2017ZA064).

\section{References}

[1] L. Yan, J. Ma, Y. Zhu et al., "miR-24-3p promotes cell migration and proliferation in lung cancer by targeting SOX7," Journal of Cellular Biochemistry, 2017.

[2] S. S. Ramalingam, T. K. Owonikoko, and F. R. Khuri, "Lung cancer: new biological insights and recent therapeutic advances," CA: A Cancer Journal for Clinicians, vol. 61, no. 2, pp. 91-112, 2011.

[3] S. Al-Saad, E. Richardsen, T. K. Kilvaer et al., "The impact of MET, IGF-1, IGF1R expression and EGFR mutations on survival of patients with non-small-cell lung cancer," PLoS ONE, vol. 12, no. 7, article e0181527, 2017.

[4] V. Ludovini, A. Flacco, and F. Bianconi, "Concomitant high gene copy number and protein overexpression of IGF1R and EGFR negatively affect disease-free survival of surgically resected non-small-cell-lung cancer patients," Cancer Chemotherapy and Pharmacology, vol. 71, no. 3, pp. 671-680, 2013.

[5] J. T. Mendell and E. N. Olson, "MicroRNAs in stress signaling and human disease," Cell, vol. 148, no. 6, pp. 1172-1187, 2012.

[6] H. Lewis, R. Lance, D. Troyer et al., "miR-888 is an expressed prostatic secretions-derived microRNA that promotes prostate cell growth and migration," Cell Cycle, vol. 13, no. 2, pp. 227-239, 2014.

[7] P. S. Chen, J. L. Su, and M. C. Hung, "Dysregulation of microRNAs in cancer," Journal of Biomedical Science, vol. 19, article 90, 2012.

[8] E. A. Elghoroury, H. G. ElDine, and S. A. Kamel, "Evaluation of miRNA-21 and miRNA Let-7 as Prognostic Markers in Patients With Breast Cancer," Clinical Breast Cancer, 2017.

[9] S. Han, J. Zhu, and Y. Zhang, "miR-144 Potentially Suppresses Proliferation and Migration of Ovarian Cancer Cells by Targeting RUNX1," Medical Science Monitor Basic Research, vol. 24, pp. 40-46, 2018.

[10] A. Li, J. Yu, H. Kim et al., "MicroRNA array analysis finds elevated serum miR-1290 accurately distinguishes patients with low-stage pancreatic cancer from healthy and disease controls," Clinical Cancer Research, vol. 19, no. 13, pp. 3600-3610, 2013.

[11] Y. Xu, Y.-F. Han, S.-J. Zhu, J.-D. Dong, and B. Ye, "MiRNA-148a inhibits cell growth of papillary thyroid cancer through STAT3 and PI3K/AKT signaling pathways," Oncology Reports, vol. 38, no. 5, pp. 3085-3093, 2017.

[12] J. Hamfjord, A. M. Stangeland, T. Hughes et al., "Differential Expression of miRNAs in Colorectal Cancer: Comparison of Paired Tumor Tissue and Adjacent Normal Mucosa Using High-Throughput Sequencing," PLoS ONE, vol. 7, no. 4, article e34150, 2012.

[13] E. Baltruskeviciene, D. Schveigert, V. Stankevicius et al., "Downregulation of miRNA-148a and miRNA-625-3p in colorectal cancer is associated with tumor budding," BMC Cancer, vol. 17, no. 1, article no. 607, 2017.

[14] M. I. Qadir and A. Faheem, "miRNA: A Diagnostic and Therapeutic Tool for Pancreatic Cancer," Critical Reviews in Eukaryotic Gene Expression, vol. 27, no. 3, pp. 197-204, 2017.

[15] Y. Wang, H. Zhao, X. Gao et al., "Identification of a threemiRNA signature as a blood-borne diagnostic marker for early diagnosis of lung adenocarcinoma," Oncotarget, vol. 7, no. 18, pp. 26070-26086, 2016.

[16] Y. K. Shi, X. Q. Liu, J. T. Lou et al., "Plasma Levels of Heat Shock Protein 90 Alpha Associated with Lung Cancer Development and Treatment Responses," Clinical Cancer Research, vol. 20, no. 23, pp. 6016-6022, 2014.

[17] R. L. Siegel, K. D. Miller, and D. V. M. Ahmedin Jemal, "Cancer statistics, 2018," CA: A Cancer Journal for Clinicians, vol. 68, no. 1, pp. 7-30, 2018.

[18] M. Usó, E. Jantus-Lewintre, R. Sirera, R. M. Bremnes, and C. Camps, "MiRNA detection methods and clinical implications in lung cancer," Future Oncology, vol. 10, no. 14, pp. 2279-2292, 2014.

[19] X. C. Wang, W. Wang, Z. B. Zhang et al., "Overexpression of miRNA-21 promotes radiation-resistance of non-small cell lung cancer," Journal of Radiation Oncology, vol. 8, article 146, 2013.

[20] Y. Y. Wang, M. Zhao, J. Y. Liu et al., "miRNA-125b regulates apoptosis of human non-small cell lung cancer via the PI3K/Akt/GSK3 beta signaling pathway," Oncology Reports, vol. 38, no. 3, pp. 1715-1723, 2017.

[21] G. D. Xu, G. F. Shao, Q. L. Pan et al., "MicroRNA-9 regulates non-small cell lung cancer cell invasion and migration by targeting eukaryotic translation initiation factor 5A2," American Journal of Translational Research, vol. 9, no. 2, pp. 478-488, 2017.

[22] C. X. Xu, H. Jin, J. Y. Shin et al., "Roles of protein kinase B/Akt in lung cancer," Frontiers in Bioscience (Elite Ed.), vol. 2, pp. 1472$1484,2010$.

[23] J. K. Yoo, H. Y. Jung, J. M. Lee et al., "The novel miR-9500 regulates the proliferation and migration of human lung cancer cells by targeting Akt1," Cell Death Differentiation, vol. 21, no. 7, pp. 1150-1159, 2014.

[24] T. J. Su, W. H. Ku, H. Y. Chen et al., "Oncogenic miR-137 contributes to cisplatin resistance via repressing CASP3 in lung adenocarcinoma," American Journal of Cancer Research, vol. 6, no. 6, pp. 1317-1330, 2016.

[25] L. H. Pearl and C. Prodromou, "Structure and mechanism of the Hsp90 molecular chaperone machinery," Annual Review of Biochemistry, vol. 75, pp. 271-294, 2006. 


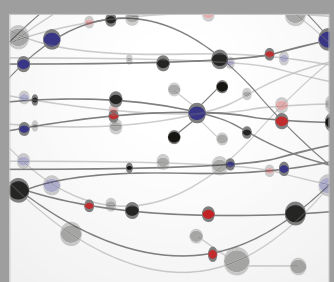

The Scientific World Journal
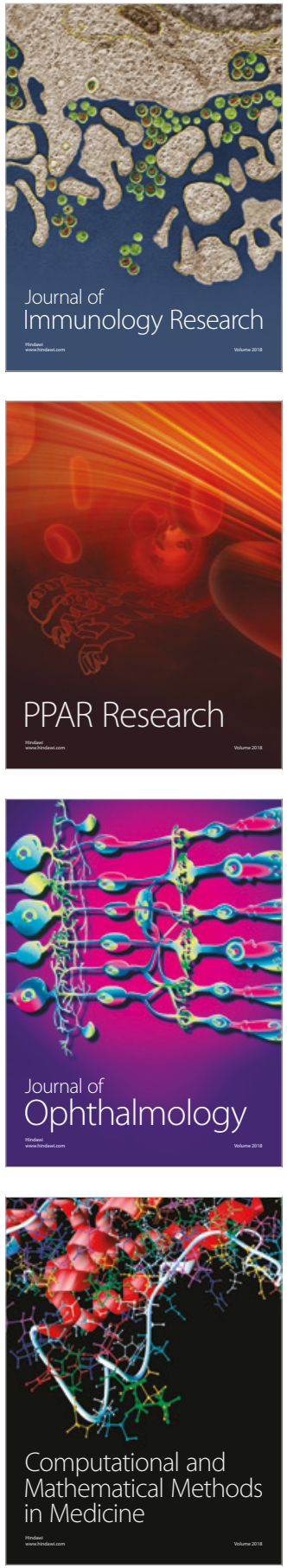



Gastroenterology Research and Practice

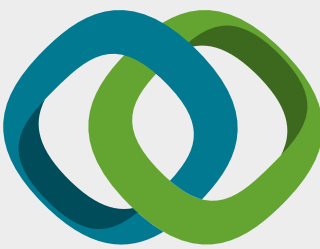

\section{Hindawi}

Submit your manuscripts at

www.hindawi.com


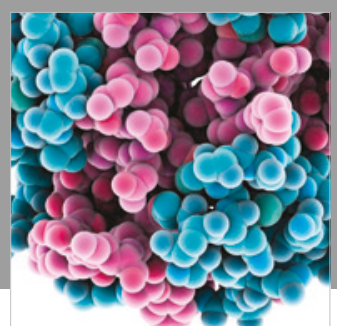

ournal of

Diabetes Research



Disease Markers
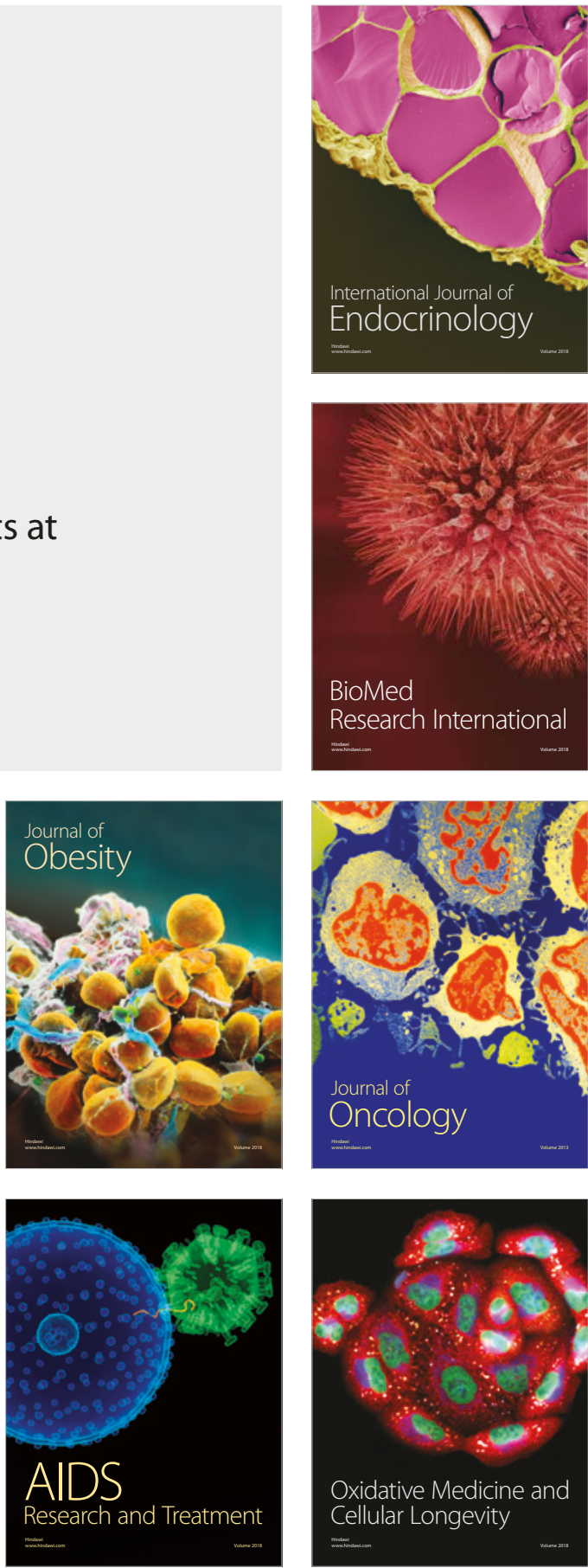